\title{
Phenomenological consequences of Higgs inflation in the NMSSM at the electroweak scale
}

\author{
Wolfgang Gregor Hollik ${ }^{\dagger a, b, c}$, Stefan Liebler ${ }^{\ddagger d}$, Gudrid Moortgat-Pick ${ }^{\diamond a, e}$, \\ Sebastian Paßehr ${ }^{*}$, Georg Weiglein ${ }^{\circ a}$ \\ ${ }^{a}$ Deutsches Elektronensynchrotron, Notkestraße 85, D-22607 Hamburg, Germany \\ ${ }^{b}$ Institute for Nuclear Physics, Karlsruhe Institute of Technology, D-76012 Karlsruhe, Germany \\ ${ }^{c}$ Institute for Theoretical Particle Physics, Karlsruhe Institute of Technology, \\ D-76128 Karlsruhe, Germany \\ ${ }^{d}$ Institute for Theoretical Physics, Karlsruhe Institute of Technology, D-76131 Karlsruhe, Germany \\ eII. Institut für Theoretische Physik, Universität Hamburg, \\ Luruper Chaussee 149, D-22761 Hamburg, Germany \\ ${ }^{f}$ Sorbonne Université, CNRS, Laboratoire de Physique Théorique et Hautes Énergies, \\ 4 Place Jussieu, F-75252 Paris CEDEX 05, France

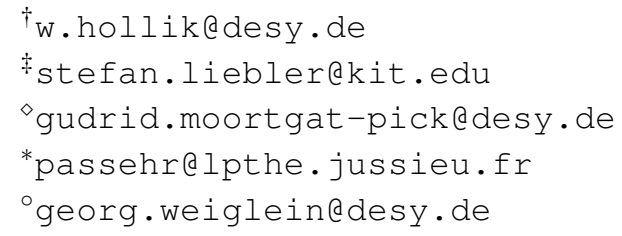

The Next-to-Minimal Supersymmetric Standard Model (NMSSM) can incorporate inflation, where a combination of the Higgs-doublet fields plays the role of the inflaton. At the high scale, the Higgs doublets are non-minimally coupled to supergravity; this coupling appears as an additional contribution to the $\mu$ term in the low-energy effective superpotential and potentially changes physics at the electroweak scale.

In a recent publication, we investigate the extended parameter space of this model with respect to collider phenomenology at the electroweak scale, and discuss scenarios which are potentially different from the pure NMSSM. We analyse the stability of the electroweak vacuum, the masses of neutralinos/charginos and Higgs bosons as well as the mixing and decays of Higgs bosons. Some important aspects of this study are described in the following.

The 39th International Conference on High Energy Physics (ICHEP2018)

4-11 July, 2018

Seoul, Korea

* Speaker. 
Introduction During the period of inflation the universe has exponentially increased its size. The idea of using the Higgs field of the Standard Model (SM) as inflaton has been proposed in Refs. [1-3]. However, the simplest implementation is fine-tuned [4]. A possible solution is given by the scale-free extension of the SM in canonical superconformal supergravity models as proposed by Refs. [5, 6] based on earlier work by Ref. [7]. In these models, two Higgs $S U(2)$-doublet superfields $\hat{H}_{u, d}$ are non-minimally coupled to Einstein gravity via $\chi \hat{H}_{u} \cdot \hat{H}_{d}$ with a dimensionless constant $\chi$; an additional Higgs singlet superfield $\hat{S}$ stabilises the potential during inflation, see Ref. [7]. These requirements can be implemented in the $\mathbb{Z}_{3}$-invariant NMSSM augmented by an additional $\mu$ term, which we call $\mu$ NMSSM in the following. In Ref. [8], we study the low-energy electroweak phenomenology of this model; studies at the scale of inflation are given in Refs. [5, 6, 9]. We have generated a model file for FeynArts [10,11], FormCalc [12] and LoopTools [12], where SARAH [13-16] has been used to generate the tree-level couplings of the $\mu$ NMSSM, and at the oneloop order we have implemented the counterterms and a renormalisation scheme that is compatible with the schemes of Refs. [17, 18] for the MSSM and NMSSM. Leading two-loop corrections from the MSSM are added with the help of FeynHiggs [19-26]. The compatibility of our scenarios with the experimental data is evaluated with the help of HiggsBounds-5.1. Obeta [27-31] and HiggsSignals-2.1.0beta [32]. In addition, we check the stability of the electroweak vacuum with respect to non-standard global minima. In view of the hints in the existing data [3335], we investigate scenarios with light Higgs singlets with production cross-sections taken from the NMSSM version of SusHi [36,37].

Analysis The superpotential of the $\mu$ NMSSM with the non-minimal coupling to supergravity reads $\mathscr{W}=\lambda \hat{S} \hat{H}_{u} \cdot \hat{H}_{d}+\frac{1}{3} \kappa \hat{S}^{3}+\frac{3}{2} m_{3 / 2} \chi \hat{H}_{u} \cdot \hat{H}_{d}$ with the gravitino mass $m_{3 / 2}$. When the singlet field acquires a vacuum expectation value (vev) $v_{s}$, the effective parameter $\mu_{\text {eff }}=\lambda v_{s}$ is generated. The value of $\chi$ can be set to about $10^{5} \lambda[6,9]$, thus that the value of $\mu=\frac{3}{2} m_{3 / 2} \chi$ is mainly steered by the gravitino mass: values of $\mu \gtrsim 1 \mathrm{TeV}$ and $\lambda \gtrsim 0.1$ require light gravitinos of $m_{3 / 2} \sim 100 \mathrm{MeV}$ (typically LSP), while for $\lambda \lesssim 10^{-5}$ the gravitino mass is similar to $\mu$. The potential cosmological gravitino problem [38], where the light gravitino dark matter overcloses the universe $[39,40]$, can be avoided by a low reheating temperature [41,42]. The Lagrangian $-\mathscr{L}_{\mathrm{s}}=\left[A_{\lambda} \lambda S H_{u} \cdot H_{d}+\frac{1}{3} A_{\kappa} \kappa S^{3}+B_{\mu} \mu H_{u} \cdot H_{d}+\right.$ h. c. $]+m_{H_{d}}^{2}\left|H_{d}\right|^{2}+m_{H_{u}}^{2}\left|H_{u}\right|^{2}+m_{s}^{2}|S|^{2}$, which softly breaks SUSY and the $\mathbb{Z}_{3}$-symmetry, is used. Further parameters which break $\mathbb{Z}_{3}$-symmetry are generated radiatively and renormalised in the $\overline{\mathrm{DR}}$ scheme at the scale of the top-mass $m_{t}$. An extensive analytic discussion of the Higgs potential and masses, vacuum stability, trilinear Higgs couplings, and neutralino, chargino and sfermion masses is given in Ref. [8]. The most important aspects are summarised in the following: (1) the Higgs mass matrix at the tree level contains terms $\propto\left(\mu+\mu_{\text {eff }}\right), \kappa \mu_{\text {eff }}$ and $\mu_{\text {eff }}^{-1}$ in the singlet-doublet mixing, and terms $\propto \kappa \mu_{\text {eff }},\left(\kappa \mu_{\text {eff }}\right)^{2}$, $\mu_{\text {eff }}^{-2}$ and $\mu / \mu_{\text {eff }}$ in the singlet elements; (2) full one-loop and leading MSSM two-loop corrections of $\mathscr{O}\left(\alpha_{t} \alpha_{s}\right)$ [43] and $\mathscr{O}\left(\alpha_{t}^{2}\right)$ [44,45] are added with the help of FeynHiggs, while further available fixed-order results [46, 47] or resummation of large logarithms [24, 25, 48] are discarded; (3) the assignment of the $C P$-even light and heavy doublets $h^{0}, H^{0}$ and singlet $s^{0}$ as well as the $C P$-odd doublet $A^{0}$ and singlet $a_{s}$ is determined from the largest admixture of the corresponding tree-level state; (4) the mass $m_{H^{ \pm}}$of the charged Higgs $H^{ \pm}$substitutes $A_{\lambda}$; (5) vacuum stability is determined from the tree-level Higgs potential to a good accuracy, see Ref. [49]; (6) metastable 

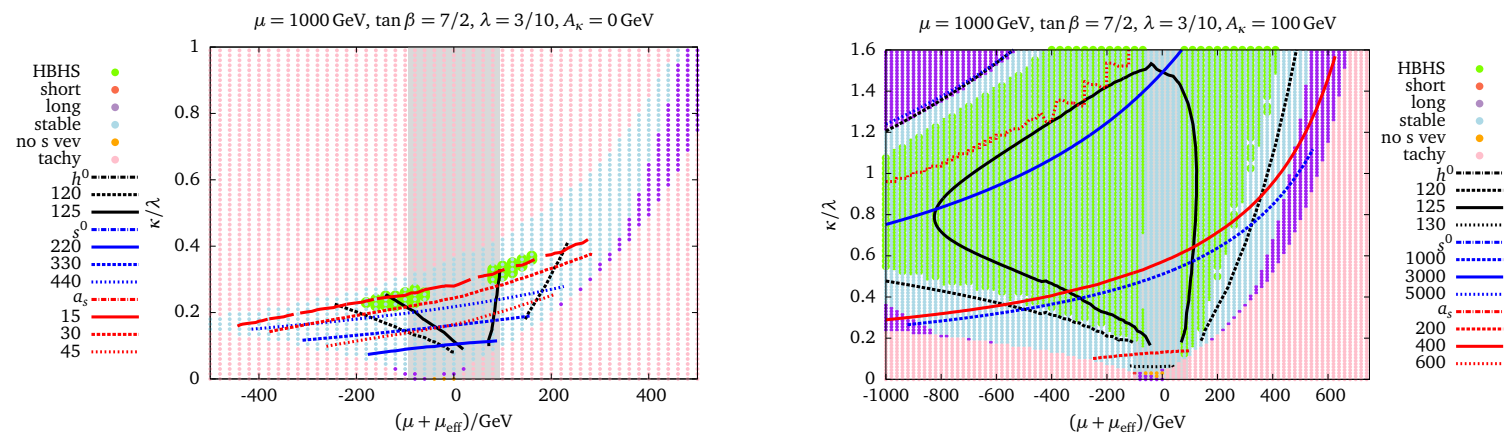

Figure 1: Mass contours of SM-like Higgs (black), $C P$-even singlet (blue) and $C P$-odd singlet (red) in the plane $\kappa / \lambda$ versus $\left(\mu+\mu_{\text {eff }}\right)$, with $\lambda=3 / 10, \mu=1000 \mathrm{GeV}$ and varying $\kappa, \mu_{\text {eff }}$ are shown. Left: $A_{\kappa}=0$, right: $A_{\kappa}=100 \mathrm{GeV}$. Furthermore, $\tan \beta=7 / 2, m_{H^{ \pm}}=800 \mathrm{GeV}, m_{t}=173.2 \mathrm{GeV}$. The soft-breaking SUSY parameters are the bilinear sfermion masses $m_{\tilde{f}}=2 \mathrm{TeV}$ and the trilinear sfermion mixings $A_{f_{3}}=4 \mathrm{TeV}$ for the third generation and $A_{f_{1,2}}=0$ for the other generations. The state of the vacuum is indicated by the background colours: stable (light blue), metastable long-lived (purple), metastable short-lived (red), tachyonic (rose). No singlet vev exists in orange regions. The green region (HBHS) is allowed by HiggsBounds and HiggsSignals. The gray-shaded area is excluded by searches for charginos at LEP [58].

vacua are classified as long- or short-lived if the tunnelling time to the deeper global minima is longer or shorter than the age of the universe; (7) constraints from charge and color-breaking minima [50-53] can be taken over to the $\mu$ NMSSM; (8) we investigate tree-level decays, but point out that loop-corrections can be large [54-57]; (9) the Higgs decays which depend on both $\mu$ and $\mu_{\text {eff }}$ are $s^{0} \rightarrow h^{0} h^{0}, H^{0} \rightarrow s^{0} h^{0}, A^{0} \rightarrow s^{0} a_{s}$ and $s^{0} \rightarrow H^{+} H^{-}$; (10) in the neutralino, chargino and sfermion sectors $\mu$ and $\mu_{\text {eff }}$ only appear as $\left(\mu+\mu_{\text {eff }}\right)$ and $\kappa \mu_{\text {eff }}$ at the tree level, which is why the shift $\mu+\mu_{\text {eff }} \rightarrow \mu_{\text {eff }}$ and rescaling $\kappa \rightarrow \tilde{\kappa}=\kappa \mu_{\text {eff }} /\left(\mu+\mu_{\text {eff }}\right)$ lead to identical spectra in the NMSSM and $\mu$ NMSSM.

Fig. 1 shows an example of our analysis in Ref. [8] for the Higgs spectrum and the vacuum stability as a function of $\kappa / \lambda$ and $\left(\mu+\mu_{\text {eff }}\right)$ with fixed $\lambda$ and $\mu$. In this setup, variations in the predicted SMlike Higgs mass are induced by admixture of the singlet or loop corrections. The value of $A_{\kappa}$ largely influences the $C P$-odd singlet mass, which explains the large tachyonic region in the left plot. In the green area at the left side the singlet admixture to the SM-like Higgs is reduced such that also the decay width for $h^{0} \rightarrow a_{s} a_{s}$ becomes smaller. The endpoints of the contours indicate a tachyonic Higgs at the tree or loop level. Each boundary is parallel to the contours of the state which turns tachyonic. The boundaries differ from the bounds of vacuum stability due to the loop corrections.

Conclusions The extended parameter space of the $\mu$ NMSSM compared to the NMSSM is investigated with respect to vacuum stability, Higgs masses and decays, and neutralino phenomenology. Scenarios with the potential to distinguish both models are proposed; differences typically occur in the singlet sector. Some excluded scenarios in the NMSSM may still be viable in the $\mu$ NMSSM.

Acknowledgments The authors are grateful to S. Abel, P. Basler, F. Domingo, K. SchmidtHoberg, T. Stefaniak and A. Westphal for helpful discussions. The authors are supported by the DFG through a lump sum fund of the SFB 676 "Particles, Strings and the Early Universe". S. P. acknowledges support by the ANR grant "HiggsAutomator" (ANR-15-CE31-0002). 


\section{References}

[1] F. L. Bezrukov, M. Shaposhnikov, Phys. Lett. B659, 703 (2008), arXiv:0710.3755.

[2] F. L. Bezrukov, A. Magnin, M. Shaposhnikov, Phys. Lett. B675, 88 (2009), arXiv:0812.4950.

[3] F. Bezrukov, M. Shaposhnikov, JHEP 07, 089 (2009), arXiv:0904.1537.

[4] J. L. F. Barbon, J. R. Espinosa, Phys. Rev. D79, 081302 (2009), arXiv:0903.0355.

[5] S. Ferrara, R. Kallosh, A. Linde, A. Marrani, A. Van Proeyen, Phys. Rev. D82, 045003 (2010), arXiv:1004.0712.

[6] S. Ferrara, R. Kallosh, A. Linde, A. Marrani, A. Van Proeyen, Phys. Rev. D83, 025008 (2011), arXiv:1008.2942.

[7] M. B. Einhorn, D. R. T. Jones, JHEP 03, 026 (2010), arXiv:0912.2718.

[8] W. G. Hollik, S. Liebler, G. Moortgat-Pick, S. Paßehr, G. Weiglein, (2018), arXiv:1809.07371.

[9] H. M. Lee, JCAP 1008, 003 (2010), arXiv:1005.2735.

[10] J. Küblbeck, M. Böhm, A. Denner, Comput. Phys. Commun. 60, 165 (1990).

[11] T. Hahn, Comput. Phys. Commun. 140, 418 (2001), arXiv:hep-ph/0012260.

[12] T. Hahn, M. Perez-Victoria, Comput. Phys. Commun. 118, 153 (1999), arXiv:hep-ph/9807565.

[13] F. Staub, Comput.Phys.Commun. 181, 1077 (2010), arXiv:0909.2863.

[14] F. Staub, Comput.Phys.Commun. 182, 808 (2011), arXiv:1002.0840.

[15] F. Staub, Computer Physics Communications 184, pp. 1792 (2013), arXiv:1207.0906.

[16] F. Staub, Comput.Phys.Commun. 185, 1773 (2014), arXiv:1309.7223.

[17] T. Fritzsche, T. Hahn, S. Heinemeyer, F. von der Pahlen, H. Rzehak, C. Schappacher, Comput. Phys. Commun. 185, 1529 (2014), arXiv:1309.1692.

[18] F. Domingo, P. Drechsel, S. Paßehr, Eur. Phys. J. C77, 562 (2017), arXiv:1706.00437.

[19] S. Heinemeyer, W. Hollik, G. Weiglein, Eur.Phys.J. C9, 343 (1999), arXiv:hep-ph/9812472.

[20] S. Heinemeyer, W. Hollik, G. Weiglein, Comput.Phys.Commun. 124, 76 (2000), arXiv:hep-ph/9812320.

[21] G. Degrassi, S. Heinemeyer, W. Hollik, P. Slavich, G. Weiglein, Eur.Phys.J. C28, 133 (2003), arXiv:hep-ph/0212020.

[22] M. Frank, et al., JHEP 0702, 047 (2007), arXiv:hep-ph/0611326.

[23] T. Hahn, S. Heinemeyer, W. Hollik, H. Rzehak, G. Weiglein, Nucl. Phys. Proc. Suppl. 205-206, 152 (2010), arXiv:1007.0956.

[24] H. Bahl, W. Hollik, Eur. Phys. J. C76, 499 (2016), arXiv:1608.01880.

[25] H. Bahl, S. Heinemeyer, W. Hollik, G. Weiglein, Eur. Phys. J. C78, 57 (2018), arXiv:1706.00346.

[26] H. Bahl, T. Hahn, S. Heinemeyer, W. Hollik, S. Paßehr, H. Rzehak, G. Weiglein, , IFT-UAM/CSIC-18-095, MPP-2018-229, to be published.

[27] P. Bechtle, O. Brein, S. Heinemeyer, G. Weiglein, K. Williams, Comput. Phys. Commun. 181, 138 (2010), arXiv:0811.4169.

[28] P. Bechtle, O. Brein, S. Heinemeyer, G. Weiglein, K. Williams, Comput. Phys. Commun. 182, 2605 (2011), arXiv:1102.1898.

[29] P. Bechtle, O. Brein, S. Heinemeyer, O. Stål, T. Stefaniak, G. Weiglein, K. Williams, PoS 156, 024 (2012), arXiv:1301.2345.

[30] P. Bechtle, et al., Eur.Phys.J. C74, 2693 (2014), arXiv:1311.0055.

[31] P. Bechtle, S. Heinemeyer, O. Stål, T. Stefaniak, G. Weiglein, Eur. Phys. J. C75, 421 (2015), arXiv:1507.06706.

[32] P. Bechtle, S. Heinemeyer, O. Stål, T. Stefaniak, G. Weiglein, JHEP 11, 039 (2014), arXiv:1403.1582.

[33] DELPHI, OPAL, ALEPH, LEP WG for Higgs Boson Searches, L3, Eur. Phys. J. C47, 547 (2006), arXiv:hep-ex/0602042.

[34] CMS collaboration, (2017), CDS 2285326.

[35] ATLAS Collaboration, CERN Report No. ATLAS-CONF-2018-025, 2018 (unpublished).

[36] R. V. Harlander, W. B. Kilgore, Phys. Rev. Lett. 88, 201801 (2002), arXiv:hep-ph/0201206.

[37] R. V. Harlander, W. B. Kilgore, Phys. Rev. D68, 013001 (2003), arXiv:hep-ph/0304035.

[38] T. Moroi, H. Murayama, M. Yamaguchi, Phys. Lett. B303, 289 (1993).

[39] H. Pagels, J. R. Primack, Phys. Rev. Lett. 48, 223 (1982).

[40] S. Weinberg, Phys. Rev. Lett. 48, 1303 (1982).

[41] J. R. Ellis, J. E. Kim, D. V. Nanopoulos, Phys. Lett. 145B, 181 (1984).

[42] M. Yu. Khlopov, A. D. Linde, Phys. Lett. 138B, 265 (1984).

[43] S. Heinemeyer, W. Hollik, H. Rzehak, G. Weiglein, Phys. Lett. B652, 300 (2007), arXiv:0705.0746.

[44] W. Hollik, S. Paßehr, Phys. Lett. B733, 144 (2014), arXiv:1401.8275.

[45] W. Hollik, S. Paßehr, JHEP 10, 171 (2014), arXiv:1409.1687.

[46] S. Paßehr, G. Weiglein, Eur. Phys. J. C78, 222 (2018), arXiv:1705.07909.

[47] S. Borowka, S. Paßehr, G. Weiglein, Eur. Phys. J. C78, 576 (2018), arXiv:1802.09886.

[48] T. Hahn, S. Heinemeyer, W. Hollik, H. Rzehak, G. Weiglein, Phys.Rev.Lett. 112, 141801 (2014), arXiv:1312.4937.

[49] W. G. Hollik, G. Weiglein, J. Wittbrodt, , DESY-18-148, to be published.

[50] U. Ellwanger, C. Hugonie, Phys. Lett. B457, 299 (1999), arXiv:hep-ph/9902401.

[51] M. Bobrowski, G. Chalons, W. G. Hollik, U. Nierste, Phys. Rev. D90, 035025 (2014), arXiv:1407.2814, [Erratum: Phys. Rev.D92,no.5,059901(2015)].

[52] W. G. Hollik, Phys. Lett. B752, 7 (2016), arXiv:1508.07201.

[53] W. G. Hollik, JHEP 08, 126 (2016), arXiv:1606.08356.

[54] D. T. Nhung, M. Mühlleitner, J. Streicher, K. Walz, JHEP 11, 181 (2013), arXiv:1306.3926.

[55] J. Baglio, C. O. Krauss, M. Mühlleitner, K. Walz, JHEP 10, 024 (2015), arXiv:1505.07125.

[56] M. Mühlleitner, D. T. Nhung, H. Ziesche, JHEP 12, 034 (2015), arXiv:1506.03321.

[57] F. Domingo, S. Heinemeyer, S. Paßehr, G. Weiglein, (2018), arXiv:1807.06322.

[58] Particle Data Group, Chin. Phys. C40, 100001 (2016). 